\title{
The Chaos Game on a General Iterated Function System
}

\author{
Michael F. Barnsley \\ Department of Mathematics \\ Australian National University \\ Canberra, ACT, Australia \\ michael . barnsley@maths . anu . edu . au \\ http://www.superfractals.com \\ Andrew Vince \\ Department of Mathematics \\ University of Florida \\ Gainesville, FL 32611-8105, USA \\ avince@ufl.edu
}

\begin{abstract}
The main theorem of this paper establishes conditions under which the "chaos game" algorithm almost surely yields the attractor of an iterated function system. The theorem holds in a very general setting, even for non contractive iterated function systems, and under weaker conditions on the random orbit of the chaos game than obtained previously.
\end{abstract}




\section{Introduction}

There are two methods for computing pictures of fractals that are attractors of iterated functions systems, the deterministic algorithm and the more efficient "chaos game" algorithm [2]. This paper concerns the chaos game on a general iterated function system (IFS) $\mathcal{F}$ defined on a complete metric space $(\mathbb{X}, d)$. The iterated function system is "general" in the following sense. The only restriction placed on $\mathbb{X}$ is that it is proper, i.e., closed balls are compact. The only restriction on the functions in $F$ is that they be continuous. In particular, they need not be contractions on $\mathbb{X}$. In fact, none of the functions needs to be a contraction on $\mathbb{X}$ with respect to any metric giving the same topology as the original metric $d$. This paper provides a natural definition of an attractor of such a general IFS. A general IFS may possess more than one attractor, one attractor, or no attractor. Examples of iterated function systems that are non contractive yet possess attractors are given in Section 4 .

The main result, Theorem 3, is new in that it shows that the chaos game algorithm can be applied to such a general IFS - in particular to situations where there is no metric, even in a neighborhood of the attractor, with respect to which the IFS is contractive. We show that the chaos game algorithm almost always yields the attractor. More precisely, if the IFS has an attractor $A$, then a random orbit, starting with a point in the basin of attraction of $A$, converges with probability one to the attractor. We show this under weaker conditions than have heretofore been described. In all other papers on this topic, of which we are aware, for example [3], 4], 8], 11], [13, [15, [19], [21], [22], it is required that the IFS be either contractive, or contractive on the average. It is also required that the process by which the functions are selected to generate the orbit is stationary and that the selection process depends Hölder continuously on the initial point; see for example [20]. For 
our result, none of these conditions are required. The only constraint on how the functions are randomly selected, one after another, is that, at each step, there is the possibility of choosing each and any of the functions, and that the probability of so doing is bounded below away from zero.

We do not ask, as is customary in descriptions of the chaos game algorithm, that the process be i.i.d. or that it be conditioned on the "past" in some restricted way. This is because we are not concerned with the existence of an invariant measure associated with a chaos game on an IFS. For such an invariant measure to exist it is typically required that an associated transition probability operator is suitably well-behaved, see [17] and references therein. Since we are only concerned with the relationship between random orbits and attractors of an IFS, we are able to obtain almost sure convergence of random orbits to attractors in very general situations.

Section 2 of this paper contains basic definitions, in particular the definition of an attractor of an IFS. Theorem 2 in Section 2 provides an expression for an attractor, of some independent interest, that will be used to prove the main result. The main result on the chaos game is Theorem 3 in Section 3. Section 4 contains examples that illustrate the practical value of Theorem 3 .

\section{General iterated function systems}

Throughout this paper $\left(\mathbb{X}, d_{\mathbb{X}}\right)$ is a complete metric space.

Definition 1 If $f_{m}: \mathbb{X} \rightarrow \mathbb{X}, m=1,2, \ldots, M$, are continuous mappings, then $\mathcal{F}=\left(\mathbb{X} ; f_{1}, f_{2}, \ldots, f_{M}\right)$ is called an iterated function system (IFS).

By slight abuse of terminology we use the same symbol $\mathcal{F}$ for the IFS,

the set of functions in the IFS, and for the following mapping. Letting $2^{\mathbb{X}}$ 
denote the collection of subsets of $\mathbb{X}$, define $\mathcal{F}: 2^{\mathbb{X}} \rightarrow 2^{\mathbb{X}}$ by

$$
\mathcal{F}(B)=\bigcup_{f \in \mathcal{F}} f(B)
$$

for all $B \in 2^{\mathbb{X}}$.

Let $\mathbb{H}=\mathbb{H}(\mathbb{X})$ be the set of nonempty compact subsets of $\mathbb{X}$. Since $\mathcal{F}(\mathbb{H}) \subset \mathbb{H}$ we can also treat $\mathcal{F}$ as a mapping $\mathcal{F}: \mathbb{H} \rightarrow \mathbb{H}$. Let $d_{\mathbb{H}}$ denote the Hausdorff metric on $\mathbb{H}$, defined in terms of $d:=d_{\mathbb{X}}$. Using the notation

$$
S+r=\{y \in \mathbb{X}: d(x, y)<r \text { for some } x \in S\}
$$

with $S \subset \mathbb{X}$ and $r>0$, a convenient definition of the Hausdorff metric (see for example [12, p.66]) is

$$
d_{\mathbb{H}}(B, C)=\inf \{r>0: B \subset C+r \text { and } C \subset B+r\}
$$

for all $B, C \in \mathbb{H}$.

A metric space $\mathbb{X}$ is locally compact if every point has a compact neighborhood and is proper if every closed ball $\{y: d(x, y) \leq r\}$ is compact. Proper spaces are locally compact, but the converse is not true in general.

Lemma 1 1. A metric space is proper if and only if $C+r$ is compact whenever $C \subset \mathbb{X}$ is compact and $r$ is a positive real number.

2. If $\mathbb{X}$ is proper, then $\mathcal{F}: \mathbb{H}(\mathbb{X}) \rightarrow \mathbb{H}(\mathbb{X})$ is continuous.

Proof: Concerning statement (1), clearly the condition on $C+r$ in the statement of the lemma implies that the metric space is proper. Just take the set $C$ to be a single point.

Conversely assume that the metric space $\mathbb{X}$ is proper. Because $C$ is compact, $C$ is totally bounded. This implies that $C$ is bounded, and hence $C+r$ 
is bounded. Therefore there is a closed ball $B$ such that $C+r \subset B$. But $B$ is compact because $\mathbb{X}$ is proper. Therefore $B$ is totally bounded. This implies that, for any $\varepsilon>0$, there is a finite set $\left\{B_{i}:=B\left(x_{i}, \varepsilon\right): i=1,2, \ldots, q\right\}$ of closed balls of radius $\varepsilon$ centered at the points $x_{i}$ such that $C+r \subset B \subset$ $\cup_{i=1}^{q} B_{i}$. Therefore $C+r$ is totally bounded. Since $C+r$ is also closed, it is compact.

Concerning statement (2), for any $B \in \mathbb{H}$, we will show that $\mathcal{F}: \mathbb{H} \rightarrow \mathbb{H}$ is continuous at $B$. Since $\mathbb{X}$ is proper and $B$ is compact, statement (1) implies that $B+1$ is compact. Therefore each $f \in \mathcal{F}$ is uniformly continuous on $B+1$. It follows that, for any $\varepsilon>0$, there is a $\delta>0$ such that $d_{\mathbb{X}}(f(x), f(y))<\varepsilon$ whenever $d_{\mathbb{X}}(x, y)<\delta$, for all $x, y \in B+1$ and for all $f \in \mathcal{F}$. Without loss of generality, take $\delta<1$.

Let $C \in \mathbb{H}$ with $d_{\mathbb{H}}(B, C)<\delta$ and let $f \in \mathcal{F}$. We will show that $d_{\mathbb{H}}(f(B), f(C))<\varepsilon$. Let $b^{\prime} \in f(B)$ and $b \in B$ such that $f(b)=b^{\prime}$. Since $d_{\mathbb{H}}(B, C)<\delta$ there is $c \in C$ such that $d(b, c)<\delta$. Since $\delta_{\varepsilon}<1$ we have $c \in$ $B+1$. It follows that $d(f(b), f(c))<\varepsilon$ and therefore that $f(B) \subset f(C)+\varepsilon$. By a similar argument $f(C) \subset f(B)+\varepsilon$. Hence $d_{\mathbb{H}}(f(B), f(C))<\varepsilon$ for all $f \in \mathcal{F}$. Finally $d_{\mathbb{H}}(\mathcal{F}(B), \mathcal{F}(C)) \leq \max _{f \in \mathcal{F}} d_{\mathbb{H}}(f(B), f(C))$.

Statement (i) of the following foundational results is well-known. A short proof can be found in [12, p.67, Theorem 2.4.4]. Statement (ii), also well known, can be found, for example, in [14]. Statement (iii) is a classical result of Hutchinson [16].

Theorem 1 (i) The metric space $\left(\mathbb{H}, d_{\mathbb{H}}\right)$ is complete.

(ii) If $\left(\mathbb{X}, d_{\mathbb{X}}\right)$ is compact then $\left(\mathbb{H}, d_{\mathbb{H}}\right)$ is compact.

(iii) If $f: \mathbb{X} \rightarrow \mathbb{X}$ is a contraction mapping for each $f \in \mathcal{F}$, then $\mathcal{F}$ : $\mathbb{H} \rightarrow \mathbb{H}$ is a contraction mapping. 
For $B \subset \mathbb{X}$, let $\mathcal{F}^{k}(B)$ denote the $k$-fold composition of $\mathcal{F}$, the union of $f_{i_{1}} \circ f_{i_{2}} \circ \cdots \circ f_{i_{k}}(B)$ over all finite words $i_{1} i_{2} \cdots i_{k}$ of length $k$. Define $\mathcal{F}^{0}(B)=B$.

Definition 2 A nonempty compact set $A \subset \mathbb{X}$ is said to be an attractor of the IFS $\mathcal{F}$ if

(i) $\mathcal{F}(A)=A$ and

(ii) there is an open set $U \subset \mathbb{X}$ such that $A \subset U$ and $\lim _{k \rightarrow \infty} \mathcal{F}^{k}(B)=A$, for all $B \in \mathbb{H}(U)$, where the limit is with respect to the Hausdorff metric.

The largest open set $U$ such that (ii) is true is called the basin of attraction for the attractor $A$ of the IFS $\mathcal{F}$.

The following observation [18, Proposition 3 (vii)], [12, p.68, Proposition 2.4.7] will be used in proving Theorem 2 .

Lemma 2 Let $\left\{B_{k}\right\}_{k=1}^{\infty}$ be a sequence of nonempty compact sets such that $B_{k+1} \subset B_{k}$ for all $k$. Then $\cap_{k \geq 1} B_{k}=\lim _{k \rightarrow \infty} B_{k}$ where convergence is with respect to the Haudorff metric.

The notation $\bar{S}$ is used to denote the closure of a set $S$, and, when $U \subset \mathbb{X}$ is nonempty, $\mathbb{H}(U)=\mathbb{H}(\mathbb{X}) \cap 2^{U}$. The quantity on the right-hand side of the equation below is sometimes called the topological upper limit of the sequence $\left\{F^{k}(B)\right\}_{k=1}^{\infty}$ and is related to other definitions of attractors of generalizations of the notion of an IFS; see for example McGehee [9] and Lesniak [18], and references in both of these. We will use Theorem 2 in the proof of Theorem 3, our main result.

Theorem 2 Let $\mathcal{F}$ be an IFS with attractor $A$, with basin of attraction $U$. If $\mathcal{F}: \mathbb{H}(U) \rightarrow \mathbb{H}(U)$ is continuous then

$$
A=\bigcap_{K \geq 1} \overline{\bigcup_{k \geq K} \mathcal{F}^{k}(B)}
$$


for all $B \subset U$ such that $\bar{B} \in \mathbb{H}(U)$.

Proof: We carry out the proof under the assumption that $B \in \mathbb{H}(U)$. It then follows from [18, Proposition 3 (i)] that Theorem 2 is true for all $B \subset U$ such that $\bar{B} \in \mathbb{H}(U)$.

Clearly $\overline{\bigcup_{k \geq K} \mathcal{F}^{k}(B)}$ is nonempty because $B$ is nonempty. We next show that $\overline{\bigcup_{k \geq K} \mathcal{F}^{k}(B)}$ is compact. Let $\left\{\mathcal{O}_{i}: i \in \mathcal{I}\right\}$ be an open cover of $\overline{\bigcup_{k \geq K} \mathcal{F}^{k}(B)}$. Since $\bigcup_{k \geq K} \mathcal{F}^{k}(\bar{B}) \subset \overline{\bigcup_{k \geq K} \mathcal{F}^{k}(B)}$ and $A=\lim _{k \rightarrow \infty} \mathcal{F}^{k}(\bar{B})$, also $\left\{\mathcal{O}_{i}: i \in \mathcal{I}\right\}$ is an open cover of $A$. Because $A$ is compact, $\left\{\mathcal{O}_{i}: i \in \mathcal{I}\right\}$ contains a finite subcollection, say $\left\{\mathcal{O}_{m}: m=1,2, . ., M\right\}$, such that $A \subset$ $\mathcal{O}:=\cup_{m=1}^{M} \mathcal{O}_{m}$. Because a metric space is normal, there is an open set $\mathcal{O}^{\prime}$ containing $A$ such that $\overline{\mathcal{O}^{\prime}} \subset \mathcal{O}$. Again using that fact that $\mathcal{F}^{k}(\bar{B})$ converges in the Hausdorff metric to $A$, there is an integer $K^{\prime}$ such that $\mathcal{F}^{k}(\bar{B}) \subset \mathcal{O}^{\prime}$ for all $k \geq K^{\prime}$. It follows that $\bigcup_{k \geq K^{\prime}} \mathcal{F}^{k}(\bar{B}) \subset \mathcal{O}^{\prime}$ and therefore $\overline{\bigcup_{k \geq K} \mathcal{F}^{k}(B)} \subset \mathcal{O}=\cup_{m=1}^{M} \mathcal{O}_{m}$ for all $K \geq K^{\prime}$. Therefore $\overline{\bigcup_{k \geq K} \mathcal{F}^{k}(B)}$ is compact if $K \geq K^{\prime}$. If $K \leq K^{\prime}$, then

$$
\overline{\bigcup_{k \geq K} \mathcal{F}^{k}(B)}=\bigcup_{K^{\prime}>k \geq K} \overline{\mathcal{F}^{k}(B)} \cup \overline{\bigcup_{k \geq K^{\prime}} \mathcal{F}^{k}(B)} .
$$

Since each function $f \in \mathcal{F}$ is continuous and $\bar{B}$ is compact, $\overline{\mathcal{F}^{k}(B)}=\mathcal{F}^{k}(\bar{B})$ is compact. Hence $\overline{\bigcup_{k \geq K} \mathcal{F}^{k}(B)}$, the finite union of compact sets on the right hand side of equation 2.1, is also compact. Since $\overline{\bigcup_{k \geq K} \mathcal{F}^{k}(B)}$ is a nonempty compact set, the nested intersection

$$
\tilde{A}:=\bigcap_{K \geq 1} \overline{\bigcup_{k \geq K} \mathcal{F}^{k}(B)}
$$

is a nonempty compact set. 
Now observe that

$$
\begin{aligned}
& \mathcal{F}(\tilde{A})=\mathcal{F}\left(\lim _{K \rightarrow \infty} \overline{\bigcup_{k \geq K} \mathcal{F}^{k}(B)}\right) \quad \text { (by Lemma 2) } \\
& =\lim _{K \rightarrow \infty} \mathcal{F}\left(\overline{\bigcup_{k \geq K} \mathcal{F}^{k}(B)}\right) \quad \text { (since } \mathcal{F}: \mathbb{H} \rightarrow \mathbb{H} \text { is continuous) } \\
& =\lim _{K \rightarrow \infty} \bigcup_{f \in \mathcal{F}} f\left(\overline{\bigcup_{k \geq K} \mathcal{F}^{k}(B)}\right) \quad \text { (by the definition of } \mathcal{F} \text { ) } \\
& =\lim _{K \rightarrow \infty} \bigcup_{f \in \mathcal{F}} \overline{f\left(\bigcup_{k \geq K} \mathcal{F}^{k}(B)\right)} \text { (since } f: \mathbb{X} \rightarrow \mathbb{X} \text { is continuous and } \\
& \overline{\cup_{k \geq K} \mathcal{F}^{k}(B)} \text { is compact) } \\
& =\lim _{K \rightarrow \infty} \overline{\bigcup_{f \in \mathcal{F}} f\left(\bigcup_{k \geq K} \mathcal{F}^{k}(B)\right)} \quad \text { (since } \bigcup_{f \in \mathcal{F}} \text { is a finite union) } \\
& =\lim _{K \rightarrow \infty} \overline{\bigcup_{k \geq K} \bigcup_{f \in \mathcal{F}} f\left(\mathcal{F}^{k}(B)\right)} \\
& =\lim _{K \rightarrow \infty} \overline{\bigcup_{k \geq K} \mathcal{F}^{k}(B)}=\tilde{A} .
\end{aligned}
$$

By an argument essentially the same as used above to show that $\overline{\bigcup_{k \geq K} \mathcal{F}^{k}(B)} \subset \mathcal{O}$, it can be shown that $\overline{\bigcup_{k \geq K} \mathcal{F}^{k}(B)}$ for $K$ sufficiently large, and hence $\tilde{A}$, lies in $U$.

Since $\tilde{A}$ is nonempty, compact and lies in $U$, the basin of attraction of $A$, we must have

$$
A=\lim _{k \rightarrow \infty} \mathcal{F}^{k}(\tilde{A})=\tilde{A}
$$

\section{The chaos game algorithm}

The following lemma will be used in the proof of Theorem 3 . 
Lemma 3 Let $\mathbb{X}$ be proper, and let $\mathcal{F}=\left(\mathbb{X} ; f_{1}, f_{2}, \ldots, f_{N}\right)$ be an IFS with attractor $A$. For any $\varepsilon>0$ there is an integer $M=M(\varepsilon)$ such that for each $x \in A+\varepsilon$ there is an integer $m=m(x, \varepsilon)<M$ such that

$$
d_{\mathbb{H}}\left(A, \mathcal{F}^{m}(\{x\})<\varepsilon / 2 .\right.
$$

Proof: Because $\mathbb{X}$ is proper and $A$ is compact, $A+\varepsilon$ is also compact by statement (1) of Lemma 1. There is no loss of generality in assuming that $\varepsilon$ is sufficiently small that $A+\varepsilon \subset U$, where $U$ is the basin of attraction of $A$. If $x \in A+\varepsilon \subset U$, then there is an integer $m(x, \varepsilon) \geq 0$ such that

$$
d_{\mathbb{H}}\left(A, \mathcal{F}^{m(x, \varepsilon)}(\{x\})<\varepsilon / 4 .\right.
$$

This is because $\lim _{k \rightarrow \infty} \mathcal{F}^{k}(\{x\})=A$.

Since $\mathbb{X}$ is proper, it follows from statement (2) of Lemma 1 that $\mathcal{F}$ : $\mathbb{H} \rightarrow \mathbb{H}$ is continuous, whence $\mathcal{F}^{m(x, \varepsilon)}: \mathbb{H} \rightarrow \mathbb{H}$ is continuous. Since $\mathcal{F}^{m(x, \varepsilon)}$ : $\mathbb{H} \rightarrow \mathbb{H}$ is continuous, there is an open ball $B\left(\{x\}, r_{x}\right)$ (in $\mathbb{H}$ ) of radius $r_{x}>0$ centered at $\{x\}$ such that $d_{\mathbb{H}}\left(\mathcal{F}^{m(x, \varepsilon)}\{x\}, \mathcal{F}^{m(x, \varepsilon)}(Y)\right)<\varepsilon / 4$ for all $Y \in B\left(\{x\}, r_{x}\right)$. It follows, in particular, that there a ball $B\left(x, r_{x}\right)$ (in $\left.\mathbb{X}\right)$ centered at $x$ such that $d_{\mathbb{H}}\left(\mathcal{F}^{m(x, \varepsilon)}\{x\}, \mathcal{F}^{m(x, \varepsilon)}(\{y\})\right)<\varepsilon / 4$ for all $y \in B\left(x, r_{x}\right)$. Combining this with equation 3.1 above gives $d_{\mathbb{H}}\left(A, \mathcal{F}^{m(x, \varepsilon)}(\{y\})\right)<\varepsilon / 2$ for all $y \in B\left(x, r_{x}\right)$.

The set of balls $\left\{B\left(x, r_{x}\right), x \in A+\varepsilon\right\}$ is an open covering of $A_{\varepsilon}$. Since $A+\varepsilon$ is compact, there is a finite subcovering $\left\{B\left(a_{i}, r_{a_{i}}\right): i=1,2, \ldots, q\right\}$ such that $A+\varepsilon \subset \cup_{i=1}^{q} B\left(a_{i}, r_{a_{i}}\right)$. If $M=\max _{i} m\left(a_{i}, \varepsilon\right)$, then for any $x \in A+\varepsilon$ there is a $i$ such that that $x \in B\left(a_{i}, r_{a_{i}}\right)$, in which case $d_{\mathbb{H}}\left(A, \mathcal{F}^{m}(\{x\})\right)<\varepsilon / 2$ for $m=m(x, \varepsilon):=m\left(a_{i}, \varepsilon\right)<M$.

Definition 3 Let $\mathcal{F}=\left(\mathbb{X} ; f_{1}, f_{2}, \ldots, f_{N}\right)$ be an IFS and $p \in(0,1 / N]$ fixed. A sequence $\left\{x_{k}\right\}_{k=0}^{\infty}$ of points in $\mathbb{X}$ is called a random orbit of $x_{0} \in \mathbb{X}$ if 
$x_{k}=f_{\sigma_{k}}\left(x_{k-1}\right), k \geq 1$ where $\sigma_{k}$ is selected randomly from $\{1,2, \ldots, N\}$, for $k=1,2, \ldots$, where the probability that $\sigma_{k}=n$ is greater than or equal to $p$, regardless of the preceeding outcomes, for all $n \in\{1,2, \ldots, N\}$ and all $k$. More formally, in terms of conditional probability,

$$
P\left(\sigma_{k}=n \mid x_{0}, \sigma_{1}, \sigma_{2}, \ldots, \sigma_{k-1}\right) \geq p \text {. }
$$

Theorem 3 Let $\mathbb{X}$ be a proper complete metric space and $\mathcal{F}=\left(\mathbb{X} ; f_{1}, f_{2}, \ldots, f_{N}\right)$ an IFS with attractor $A$ and basin of attraction $U$. If $\left\{x_{k}\right\}_{k=0}^{\infty}$ is a random orbit of $x_{0} \in U$ under $\mathcal{F}$, then with probability one,

$$
A=\lim _{K \rightarrow \infty}\left\{x_{k}\right\}_{k=K}^{\infty}
$$

where the limit is with respect to the Hausforff metric.

Proof: We first claim that, given any $\varepsilon>0$, there is an integer $K>0$ such that

$$
x_{k} \in A+\varepsilon
$$

for all $k \geq K$. Since $\mathbb{X}$ is proper, $\mathcal{F}$ is continuous by Theorem 1 , By Theorem 2 and Lemma 2 ,

$$
A=\lim _{L \rightarrow \infty} \overline{\bigcup_{j \geq L} \mathcal{F}^{j}\left(\left\{x_{0}\right\}\right)} .
$$

It follows that, for any $\varepsilon>0$, we can choose $K$ so that

$$
x_{k} \in \overline{\bigcup_{j \geq K} \mathcal{F}^{j}\left(\left\{x_{0}\right\}\right)} \subset A+\varepsilon
$$

for all $k \geq K$, as claimed.

We next show that, for any $\varepsilon>0$, there is an integer $K>0$ such that

$$
d_{\mathbb{H}}\left(A,\left\{x_{k}\right\}_{k=L}^{\infty}\right)<\varepsilon
$$


with probability one, for all $L \geq K$. This is equivalent to the statement of the theorem. To prove this, let $\varepsilon>0$. If $K$ is as specified in the paragraph above, then by (3.2) we have $x_{L} \in A+\varepsilon$ for $L \geq K$. The attractor $A$, being compact, is totally bounded. Let $\left\{a_{q}: i=1,2, \ldots, Q\right\}$ be a set of points such that $A \subset \cup_{q=1}^{Q} B\left(a_{i}, \varepsilon / 2\right)$, where $B\left(a_{q}, \varepsilon / 2\right)$ is the ball of radius $\varepsilon / 2$ centered at $a_{q}$. Note that each $a_{q}$ and $Q$ depend on $\varepsilon$. By Lemma 3 there is an integer $M$ such that, for each $x \in A+\varepsilon$, there is $m<M$ such that $d_{\mathbb{H}}\left(A, \mathcal{F}^{m}(\{x\})<\varepsilon / 2\right.$. Hence

$$
d_{\mathbb{H}}\left(A, \mathcal{F}^{m}\left(\left\{x_{L}\right\}\right)\right)<\varepsilon / 2
$$

for some integer $m<M$. Therefore there is a sequence of symbols $\sigma_{L+1} \sigma_{L+2} \ldots \sigma_{L+m}$ such that $f_{\sigma_{L+m}} \circ f_{\sigma_{L+m-2}} \circ \ldots \circ f_{\sigma_{L+1}}\left(x_{L}\right) \in B\left(a_{1}, \varepsilon / 2\right)$. (We adopt the convention that the composition on the left equals $x_{L}$ if $m=0$.) It follows that

$$
B\left(a_{1}, \varepsilon / 2\right) \bigcap\left\{x_{k}\right\}_{k=L}^{L+M-1} \neq \emptyset,
$$

or

$$
B\left(a_{1}, \varepsilon / 2\right) \subset\left\{x_{k}\right\}_{k=L}^{L+M-1}+\varepsilon .
$$

The probability that this event occurs, i.e., that the particular sequence $\sigma_{L+1} \sigma_{L+2} \ldots \sigma_{L+m}$ is chosen, is greater than $p^{M}$. By repeating this argument, we deduce that the probability that

$$
B\left(a_{q}, \varepsilon / 2\right) \subset\left\{x_{k}\right\}_{k=L+(q-1) M}^{L+q M-1}+\varepsilon
$$

is greater than $p^{M}>0$, for each $q \in\{1,2, \ldots, Q\}$, regardless of whether or not the preceding events occur. (That is not to say that the events are independent.) It follows that the probability that all of these events occur is greater than $p^{Q M}$. Consider the event $E_{1}$ defined by

$$
\bigcup_{q=1}^{Q} B\left(a_{q}, \varepsilon / 2\right) \nsubseteq\left\{x_{k}\right\}_{k=L}^{L+Q M-1}+\varepsilon
$$


The probability of $E_{1}$ is less than $\left(1-p^{Q M}\right)$. By a similar argument the probability of the event $E_{r}, r \geq 1$, defined by

$$
\bigcup_{q=1}^{Q} B\left(a_{q}, \varepsilon / 2\right) \nsubseteq\left\{x_{k}\right\}_{k=L+(r-1) Q M}^{L+r Q M-1}\left\{x_{k}\right\}+\varepsilon
$$

is less than $\left(1-p^{Q M}\right)$, regardless of whether or not the previous events $E_{1}, E_{2}, \ldots, E_{r-1}$ occurred, for $r=2,3, \ldots$ It follows that the probability of the event $E_{1} \cap E_{2} \cap \cdots \cap E_{r}$ is less than $\left(1-p^{Q M}\right)^{r}$, for all $r=1,2, \ldots$ This inequality holds regardless of the fact that the $E_{r}$ are not independent. To simplify notation, let $s=\left(1-p^{Q M}\right)<1$ so that

$$
\operatorname{pr}\left(E_{1} \cap E_{2} \cap \cdots\right) \leq \operatorname{pr}\left(E_{1} \cap E_{2} \cap \cdots \cap E_{r}\right)=s^{r}
$$

for all $r$. This implies that $\operatorname{pr}\left(\cap_{r=1}^{\infty} E_{r}\right)=0$. Hence, with probability one, there is an $R$ such that

$$
\bigcup_{q=1}^{Q} B\left(a_{q}, \varepsilon / 2\right) \subset\left\{x_{k}\right\}_{k=L+(R-1) Q M}^{L+R Q M-1}+\varepsilon .
$$

Since $A \subset \cup_{q=1}^{Q} B\left(a_{q}, \varepsilon / 2\right)$ it follows that, with probability one, there is $R$ such that

$$
A \subset\left\{x_{k}\right\}_{k=L+(R-1) Q M}^{L+R Q M-1}+\varepsilon .
$$

Because $L$ is an arbitrary integer greater than or equal to $K$,

$$
A \subset\left\{x_{k}\right\}_{L}^{\infty}+\varepsilon .
$$

for any $L \geq K$. But by (3.2) we also have

$$
\left\{x_{k}\right\}_{k=L}^{\infty} \subset A+\varepsilon .
$$

for any $L \geq K$. Hence, with probability one

$$
d_{\mathbb{H}}\left(A,\left\{x_{k}\right\}_{k=L}^{\infty}\right)<\varepsilon .
$$


for any $L \geq K$.

It follows that

$$
\bigcap_{K \geq 1} \overline{\left\{x_{k}\right\}_{k=K}^{\infty}}=\bigcap_{K \geq 1} \overline{\bigcup_{k \geq K} \mathcal{F}^{k}(B)}
$$

almost surely, for $x_{0} \in U$ and $B \in \mathbb{H}(U)$, for example, $B=\left\{x_{0}\right\}$. We draw attention to this equality because it seems suprising when $\mathcal{F}$ contains for than one function; the set $\left\{x_{k}\right\}_{K}^{\infty}$ seems sparse in comparison to $\bigcup_{k \geq K} \mathcal{F}^{k}(B)$.

\section{Examples}

Example 1 The IFS $\mathcal{F}$ in this example has a unique attractor, yet each $f \in \mathcal{F}$ fails to be a contraction with respect to any metric giving the same topology as the original metric. With probability one, the chaos game applied to this example "draws a picture" of the attractor of the IFS.

If $\mathbb{X}=\left\{(x, y) \in \mathbb{R}^{2}: x^{2}+y^{2}=1\right\}$ and $d$ is the Euclidean metric, then $(\mathbb{X}, d)$ is a compact metric space. Let $\mathcal{F}=\left(\mathbb{X} ; f_{1}, f_{2}\right)$ where $f_{1}(x, y)=(x, y)$ and $f_{2}(x, y)=(x \cos \alpha-y \sin \alpha, x \sin \alpha+y \cos \alpha)$, where $\alpha / \pi$ is irrational. The map $f_{1}$ is the identity map and $f_{2}$ is a rotation through angle $\alpha$ anticlockwise about the origin. Since neither $f_{1}$ nor $f_{2}$ has a unique fixed point, it follows that there exists no metric $\tilde{d}$ on $\mathbb{X}$ such that $(\mathbb{X}, \tilde{d})$ is complete and either $f_{1}$ or $f_{2}$ is a contraction. On the other hand, $\mathcal{F}$ has a unique attractor $A=\mathbb{X}$. To see this, first note that $\mathcal{F}(\mathbb{X})=\mathbb{X}$. Also, if $\left(x_{0}, y_{0}\right) \in \mathbb{X}$ then

$$
\mathcal{F}^{k}\left(x_{0}, y_{0}\right)=\left\{f_{2}^{j}\left(x_{0}, y_{0}\right)\right\}_{j=0}^{k}
$$

for all $k$. The right hand side is well known to converge in the Hausdorff metric, as $k$ tends to infinity, to $\mathbb{X}$. If follows that $\mathcal{F}^{k}(B)$ converges to $\mathbb{X}$ for all $B \in \mathbb{H}(\mathbb{X})$. By definition 2 , the IFS $\mathcal{F}$ has a unique attractor, namely $\mathbb{X}$. 
By Theorem 3, with probability one, the chaos game applied to this example "draws a picture" of the unit circle, the attractor of the IFS.

Example 2 In the same spirit as Example1, this is an IFS on the real projective plane that possesses an attractor, but there is no equivalent metric with respect to which the maps of the IFS are contractive. Again, with probability one, the chaos game "draws a picture" of the attractor of the IFS.

This example appears in [1]. The metric space is $\left(\mathbb{R} P^{2}, d\right)$, where $\mathbb{R} P^{2}$ denotes real projective two-dimensional space, and $d$ denotes the round metric; see [1]. Let $\mathcal{F}=\left(\mathbb{R} P^{2} ; f_{1}, f_{2}\right)$, where the pair of projective transformations $f_{1}$ and $f_{2}$ are represented (acting on homogeneous coordinates), respectively, by the pair of matrices

$$
\left(\begin{array}{lll}
1 & 0 & 0 \\
0 & 2 & 0 \\
0 & 0 & 2
\end{array}\right) \quad \text { and } \quad\left(\begin{array}{ccc}
1 & 0 & 0 \\
0 & 2 \cos \alpha & -2 \sin \alpha \\
0 & 2 \sin \alpha & 2 \cos \alpha
\end{array}\right) \text {, }
$$

and $\alpha / \pi$ is irrational. In terms of homogeneous coordinates $(x, y, z)$, the attractor of $\mathcal{F}$ is the line $x=0$. This can be proved by a similar argument to the one in Example 1.

Such non-contractive IFSs occur often in real projective IFS theory. Theorem 3 tells us that the chaos game algorithm can always be applied to compute approximate pictures of attractors of real projective IFSs.

Example 3 This is a superfractal example.

Let $\left(\mathbb{X}, d_{\mathbb{X}}\right)$ be a compact metric space. Consider the IFS $\mathbb{F}=\left(\mathbb{H}(\mathbb{X}) ; \mathcal{F}_{1}, \mathcal{F}_{2}\right)$ where the mappings $\mathcal{F}_{i}: \mathbb{H}(\mathbb{X}) \rightarrow \mathbb{H}(\mathbb{X})$ are the maps corresponding to the IFSs $\mathcal{F}_{1}=\left(\mathbb{X} ; f_{11}, f_{12}\right)$ and $\mathcal{F}_{2}=\left(\mathbb{X} ; f_{21}, f_{22}\right)$ - as explained directly after definition 1 . The space $\mathbb{H}(\mathbb{X})$ is endowed with the Hausdorff 
metric $d_{\mathbb{H}(\mathbb{X})}$ derived from the Euclidean metric $d_{\mathbb{X}}$ on $\mathbb{X}$. In particular, since the functions $f_{i j}$ are continuous, the mappings $\mathcal{F}_{i}: \mathbb{H}(\mathbb{X}) \rightarrow \mathbb{H}(\mathbb{X})$ are indeed continuous and $\mathbb{F}$ is a well-defined IFS. By Theorem $\mathbb{1},\left(\mathbb{H}(\mathbb{X}), d_{\mathbb{H}(\mathbb{X})}\right)$ is a compact, and hence normal, metric space. Again invoking Theorem 1, it follows that $\left(\mathbb{H}(\mathbb{H}(\mathbb{X})), d_{\mathbb{H}(\mathbb{H}(\mathbb{X}))}\right)$ is also a compact, and hence normal metric space. It follows that $\mathbb{F}: \mathbb{H}(\mathbb{H}(\mathbb{X})) \rightarrow \mathbb{H}(\mathbb{H}(\mathbb{X}))$ defined by

$$
\mathbb{F}(\mathbb{B})=\mathcal{F}_{1}(\mathbb{B}) \cup \mathcal{F}_{2}(\mathbb{B})
$$

for all $\mathbb{B} \in \mathbb{H}(\mathbb{H}(\mathbb{X}))$. is continuous with respect to the Hausdorff-Hausdorff metric $d_{\mathbb{H}(\mathbb{H}(\mathbb{X}))}$, where

$$
\mathcal{F}_{i}(\mathbb{B})=\left\{\mathcal{F}_{i}(B): B \in \mathbb{B}\right\} \subset \mathbb{H}(\mathbb{H}(\mathbb{X}))
$$

for all $\mathbb{B} \in \mathbb{H}(\mathbb{H}(\mathbb{X}))$. Consistent with the terminology in [5], [6], [7], an attractor of such an IFS $\mathbb{F}$ is called a superfractal. A superfractal thus consists of a set $\mathbb{A}$ whose elements are compact subsets of $\mathbb{X}$. The set $\mathbb{A}$ is itself compact in the Hausdorff-Hausdorff metric. Theorem 3 tells us that such an attractor can be computed by means of the chaos game, regardless of whether or not there exists a metric such that the constituent mappings are contractive. Many examples of approximations to sets belonging to superfractals, calculated using the chaos game algorithm, are illustrated in [5]. These calculations were made, without any special care over whether or not the random selections were precisely i.i.d. or even whether or not the constituent IFS's were contractive. This paper justifies such apparent carelessness.

\section{References}

[1] M. F. Barnsley, A. Vince, D. C. Wilson, Real projective iterated function systems, Preprint (2010). 
[2] M. F. Barnsley, Fractals Everywhere, Academic Press, Boston, MA, 1988.

[3] M. F. Barnsley and S. G. Demko, Iterated function systems and the global construction of fractals, Proc. Roy. Soc. London Ser. A 399 (1985) 243-275.

[4] M. F. Barnsley, S. G. Demko, J. H. Elton, J. S. Geronimo, Invariant measures arising from iterated function systems with place-dependent probabilities, Ann. Inst. H. Poincaré, 24 (1988) 367-394.

[5] Michael F. Barnsley, Superfractals, Cambridge University Press, Cambridge, 2006.

[6] M. F. Barnsley, J. Hutchinson, Ö. Stenflo, V-variable fractals: fractals with partial self similarity, Advances in Mathematics, 218 (2008) 20512088.

[7] M. F. Barnsley, J. Hutchinson, Ö. Stenflo, $V$-variable fractals, dimension results, Forum Mathematicum, to appear.

[8] Marc A. Berger, Random affine iterated function systems: curve generation and wavelets, SIAM Review, 34 (1992) 361-385.

[9] R. P. McGehee, T. Wiandt, Conley decomposition for closed relations, Difference Equations and Applications, 12 (2006) 1-4\%.

[10] R. M. Dudley, Real Analysis and Probability, Wadsworth \& Brooks/Cole, Pacific Grove, California, 1989.

[11] J. H. Elton, An ergodic theorem for iterated maps, Ergodic Theory Dynam. Systems, 7 (1987) 481-488. 
[12] Gerald A. Edgar, Measure, Topology, and Fractal Geometry, SpringerVerlag, New York, 1990.

[13] Joanna Jaroszewska, Iterated function systems with continuous place dependent probabilities, Universitatis Iagellonicae Acta Mathematica, 40 (2002) 137-146.

[14] Jeff Henrikson, Completeness and total boundednesss of the Hausdorff metric, MIT Undergraduate Journal of Mathematics, 1 (1999) 69-79.

[15] B. Forte and F. Mendivil, A classical ergodic property for IFS: A simple proof, Ergodic Theory Dynam. Systems 18 (1998) 609-611.

[16] J. E. Hutchinson, Fractals and self-similarity, Indiana Univ. Math. J. 30 (1981) 713-747.

[17] Benton Jamison, Asymptotic stability of successive iterates of continuous functions under a Markov operator, Journal of mathematical analysis and applications, 9 (1964) 203-214.

[18] Krzysztof Leśniak, Stability and invariance of multivalued iterated function systems, Math. Slovaca, 53 (2003) 393-405.

[19] O. Onicescu and G. Mihok, Sur les chaînes de variables statistiques, Bull. Sci. Math. de France 59 (1935) 174-192.

[20] Ö Stenflo, Uniqueness of invariant measures for place-dependent random iterations of functions, in Fractals in Multimedia, M. F. Barnsley, D. Saupe, and E. R. Vrscay, eds., IMA Volumes in Mathematics and its Applications, vol. 132, Springer-Verlag, New York, 2002.

[21] Ivan Werner, Ergodic theorem for contractive Markov systems, Nonlinearity, 17 (2004) 2303-2313. 
[22] Robert Scealy, V-variable fractals and interpolation, Ph.D. Thesis, Australian National University, 2008. 